\title{
Positive and Negative Psychosocial Impact of Being Diagnosed With Cancer as an Adolescent or Young Adult
}

\author{
Keith M. Bellizzi, PhD, MPH'; Ashley Smith, PhD, $\mathrm{MPH}^{2}$; Steven Schmidt, MA'; Theresa H. M. Keegan, PhD ${ }^{3,4}$; \\ Brad Zebrack, PhD, MSW, MPH${ }^{5}$; Charles F. Lynch, MD, PhD ${ }^{6}$; Dennis Deapen, $\mathrm{DrPH}^{7}$; Margarett Shnorhavorian, MD, MPH ${ }^{8}$; \\ Bradley J. Tompkins, MS, $\mathrm{MPH}^{9}$; Michael Simon, $\mathrm{MD}^{10}$; and the Adolescent and Young Adult Health Outcomes and Patient \\ Experience (AYA HOPE) Study Collaborative Group
}

BACKGROUND: The objective of this study was to explore the psychosocial impact of cancer on newly diagnosed adolescent and young adult (AYA) cancer patients. METHODS: This was a population-based, multicenter study of 523 newly diagnosed AYA survivors (ages 15-39 years) of germ cell cancer $(n=204)$, non-Hodgkin lymphoma $(n=131)$, Hodgkin lymphoma $(n=142)$, acute lymphocytic leukemia $(n=21)$, or sarcoma $(n=25)$ from 7 National Cancer Institute Surveillance, Epidemiology, and End Results (SEER) registries. Age at diagnosis was categorized into 3 groups (ages 15-20 years, 21-29 years, and 30-39 years). RESULTS: Respondents (43\% response rate), on average ( \pm standard deviation), were aged $29=6.7$ years, and most patients (80.1\%) were not receiving treatment at the time the completed the survey. With modest differences between the age groups, the most prevalent areas of life impacted in a negative way were financial, body image, control over life, work plans, relationship with spouse/significant other, and plans for having children. Endorsement of positive life impact items also was evident across the 3 age groups, particularly with regard to relationships, future plans/goals, and health competence. CONCLUSIONS: The current results indicated that there will be future need for interventions targeting financial assistance, body image issues, relationships, and helping AYAs to attain their education objectives. Cancer 2012;118:5155-62. (C) 2012 American Cancer Society.

KEYWORDS: adolescent and young adult cancer, psychosocial impact, oncology, quality of life.

\section{INTRODUCTION}

Adolescents and young adults (AYAs) are in a challenging period of psychosocial development as they navigate the many tasks associated with this phase of life, such as education and career attainment, dating, family relationships, and developing a healthy body image. ${ }^{1,2}$ A diagnosis of cancer as an AYA can significantly disrupt or delay achieving these developmental milestones. ${ }^{3,4}$ At the same time, there is the potential for positive, life-affirming experiences as a result of a diagnosis of cancer as an AYA. ${ }^{5,6}$ Studies of pediatric cancer survivors and older adults with cancer clearly demonstrate that cancer and its treatment can affect a variety of life domains, including work, education, and family. ${ }^{7-11}$ However, little is known about the negative and positive psychosocial impact of being diagnosed with cancer as an AYA, a time of profound development and maturation.

In 2005, the National Cancer Institute (NCI) partnered with LiveStrong to conduct a progress review group that would address the research and cancer care needs of AYAs (ages 15-39 years). ${ }^{12}$ This resulted in a report that provided specific recommendations, including the need to 1) identify the characteristics that distinguish the unique cancer burden in AYA patients, and 2) create the tools to study AYA cancers. Despite these recommendations, the majority of extant research has focused on biologic correlates of health outcomes or strategies to increase enrollment in cancer clinical trials

Corresponding author: Keith M. Bellizzi, PhD, MPH, Department of Human Development and Family Studies, University of Connecticut, 348 Mansfield Road, Storrs, CT 06269; Fax: (860) 486-0663; keith.m.bellizzi@uconn.edu

The Adolescent and Young Adult Health Outcomes and Patient Experience (AYA HOPE) Study Collaborative Group: California Cancer Registry/Public Health Institute, Sacramento, Calif (Rosemary Cress, DrPH [principal investigator]; Gretchen Agha; and Mark Cruz); Fred Hutchinson Cancer Research Center, Seattle, Wash (Stephen M. Schwartz, PhD [principal investigator]; Martha Shellenberger; and Tiffany Janes); Karmanos Cancer Center, Detroit, Mich (Ikuko Kato, PhD [principal investigator]; Ann Bankowski; and Marjorie Stock); Louisiana State University, New Orleans, La (Xiao-Cheng Wu, MD, MPH [principal investigator]; and Vivien Chen, PhD); Bradley Tompkins Cancer Prevention Institute of California, Fremont, Calif (Theresa Keegan, PhD, MS [principal investigator]; Laura Allen; and Zinnia Loya); Karen Hussain University of lowa, lowa City, lowa (Charles F. Lynch, MD, PhD [principal investigator]; Michele M. West, PhD; Lori A. Odle, RN); University of Southern California, Los Angeles, Calif (Ann Hamilton, PhD [principal investigator]; Jennifer Zelaya; Mary Lo; and Urduja Trinidad); National Cancer Institute, Bethesda, Md (Linda C. Harlan, BSN, MPH, PhD [investigator]; Ashley Wilder Smith, PhD, MPH [coinvestigator]; Sonja M. Stringer, MPH; Gretchen Keel, BS, BA). Consultants: Arnold Potosky, PhD; Keith Bellizzi, PhD, MPH; Karen Albritton, MD; Michael Link, MD; and Brad Zebrack, PhD.

${ }^{1}$ Department of Human Development and Family Studies, University of Connecticut, Storrs, Connecticut; ${ }^{2}$ Outcomes Research Branch, National Cancer Institute, Bethesda, Maryland; ${ }^{3}$ Cancer Prevention Institute of California, Fremont, California; ${ }^{4}$ Department of Health Research and Policy, Stanford University School of Medicine, Stanford, California; ${ }^{5}$ University of Michigan School of Social Work, Ann Arbor, Michigan; ${ }^{6}$ Department of Epidemiology, The University of lowa, lowa City, lowa; ${ }^{7}$ Department of Preventive Medicine, University of Southern California, Los Angeles, California; ${ }^{8}$ Pediatric Urology, Seattle Children's Hospital, Seattle, Washington; ${ }^{9}$ Vermont Department of Health, Burlington, Vermont; ${ }^{10}$ Barbara Ann Karmanos Cancer Institute, Detroit, Michigan

DOI: 10.1002/cncr.27512, Received: October 19, 2011; Revised: January 3, 2012; Accepted: January 23, 2012, Published online March 13, 2012 in Wiley Online Library (wileyonlinelibrary.com) 
for patients in this age group, ${ }^{13,14,15}$ with less attention given to the psychosocial impact of being diagnosed during adolescence and young adulthood.

In a study of body image and social adjustment in 21 adolescents with cancer and healthy controls, body image was rated more negatively in cancer patients, and this negative rating was more pronounced with longer time after treatment. ${ }^{16}$ Another study of health care needs of 37 patients with cancer ages 18 to 44 years suggested that patients desire treatment decisions that take into account the risks to successfully achieving the developmental tasks that face this population, such as education, family planning, career development, and employment. ${ }^{17}$ A study of health and supportive care needs of 1088 AYAs (ages 18-39 years) with cancer indicated that having adequate health insurance, support from family and friends, and information about fertility were the most salient for this group. ${ }^{18}$ Finally, a qualitative study of 14 young adults (ages 14-22 years) recently diagnosed with cancer identified family support, belief in God, and belief in one's own personal resources as the most important sources of support. ${ }^{19}$ However, although these studies have added to the almost nonexistent body of knowledge with regard to psychosocial issues of being diagnosed with cancer as an AYA, the generalizability of these findings are limited by convenience sampling and small sample sizes.

To address some of the gaps in the literature, the Adolescent and Young Adult Health Outcomes and Patient Experience (AYA HOPE) Study ${ }^{20}$ was formed. AYA HOPE used data from the NCI's Surveillance, Epidemiology and End Results (SEER) population-based registries to determine the feasibility of collecting patient consent, medical records, and survey data from recently diagnosed AYA cancer patients in population-based community settings. The objectives of the current article were 1) to identify the negative and positive impact of cancer on developmental aspects of adolescence and young adulthood, and 2) to examine these impacts according to stage of development (age at diagnosis). Understanding psychosocial issues from a developmental perspective can inform the development of age-appropriate interventions that promote adjustment and coping.

\section{MATERIALS AND METHODS}

\section{Participants and Procedures}

Eligible AYA cancer patients were selected from 7 SEER registries if they were 1) ages 15 to 39 years at the time they were diagnosed with germ cell cancer, non-Hodgkin lymphoma (NHL), Hodgkin lymphoma (HL), acute lymphocytic leukemia (ALL), or sarcoma (Ewing, osteosarcoma, or rhabdomyosarcoma) between July 1, 2007 and October 31, 2008, 2) <14 months postdiagnosis; 3) residents of the study area; and 4) able to read English. The SEER registries used were Detroit, Seattle/Puget Sound, Los Angeles County, San Francisco/Oakland, Greater California (13 counties around Sacramento plus Orange County), and the states of Iowa and Louisiana. Institutional review board approval was obtained for the 7 registries and for the NCI. Recruitment details and methodology have been published elsewhere. ${ }^{20}$

Of 1405 patients who were identified as potentially eligible, physicians refused access to 6 patients, 11 patients were identified as dead by their physician, 70 patients exceeded their 14-month eligibility date before contact, and 9 patients were ineligible. Eligible patients $(\mathrm{n}=$ 1309) were mailed a cover letter; a brochure introducing the study; an overall study consent document; a paper survey; a web address for the online survey; a preaddressed, stamped return envelope for study materials; a LIVESTRONG bracelet; and notification that they would receive $\$ 50$ for completing and returning the survey. If the patient was a minor at the time of contact, the study materials were mailed to the parent/guardian and included a consent document requiring signatures from the parent/guardian and a signature of assent from the minor. Of the 1309 patients who were mailed the survey, 85 were further identified as ineligible, because they did not speak English or denied they had cancer, and 16 additional patients had died. In total, 523 patients completed the survey: $22.5 \%$ of surveys were completed online, $2 \%$ were completed by telephone, and $76 \%$ were completed by hard copy.

\section{Measures}

The AYA HOPE patient survey (available at: http://outcomes.cancer.gov/surveys/aya/ [accessed January 2, 2012]) included questions about sociodemographic characteristics, various barriers to and quality of health care, treatment and symptoms, insurance status, information and service needs, psychosocial impact of cancer, and quality of life. Because this report is 1 in a series of articles from the AYA HOPE study, the section below describes only the variables that were used in the current analysis.

\section{Demographic and disease characteristics}

Sociodemographic information included sex, age, race, education, and marital status. Age at diagnosis was categorized into 3 groups (ages 15-20 years, 21-29 years, and 30-39 years) to examine impact at different developmental periods. Disease characteristics included cancer type, treatment status (on or off treatment), and type of 
treatment received. Treatment status was obtained by patient survey, and cancer type and type of treatment received were obtained from medical records and the SEER cancer registry database.

\section{Life impact}

To identify the positive and negative psychosocial impact of cancer, participants completed a modified version of the 18-item Life Impact Checklist. ${ }^{7,21}$ Nine of the 18 items were from the original checklist that was used in a study of breast cancer survivors, ${ }^{21}$ and 9 items related to other life domains (body image, future goal setting, plans for education and work) were included that have been identified as important to AYA survivors of cancer. ${ }^{18,22}$ Individuals were asked to indicate the kind of overall impact cancer had on specific areas of their life based on the checklist. Response choices were based on a 6-point scale, and the choices were categorized as 0 (does not apply), 1 (very negative), 2 (somewhat negative), 3 (no impact), 4 (somewhat positive), and 5 (very positive).

\section{Data Analysis}

Descriptive analyses, including frequencies and percentages, are reported to describe the study respondents. To describe the impact of cancer on respondents, frequencies and percentages (positive, negative, no impact) are presented for the sample stratified by age group. Like previous research that used this impact checklist, ${ }^{7}$ the very negative and somewhat negative response choices were collapsed into a single negative category. Similarly, very positive and somewhat positive response choices were combined to create a positive category. Percentages reported exclude the does not apply response option from the denominator, so that relevant items can be compared across age groups. Chi-square tests were conducted to examine statistically significant differences among each impact item comparing the younger age group (ages 15-20 years) and the older age group (ages 30-39 years) with the middle age group (ages 21-29 years).

\section{RESULTS}

\section{Demographic and Disease Characteristics of Respondents}

The final sample for analyses included 523 AYA survivors (43\% response rate) of germ cell cancer $(n=204)$, NHL $(\mathrm{n}=131)$, HL $(\mathrm{n}=142)$, ALL $(\mathrm{n}=21)$, and sarcoma $(\mathrm{n}=25)$. The average age of respondents ( \pm standard deviation) was $29 \pm 6.7$ years (Table 1 ).

Analyses of responders versus nonresponders suggest that females were more likely to participate $(P<.0001)$, and non-Hispanic black patients $(P<.05)$ and Hispanic

Table 1. Demographic and Disease Characteristics of Adolescent and Young Adult Cancer Patients in the Adolescent and Young Adult Health Outcomes and Patient Experience Study $(n=523)$

\section{Variable}

Sex

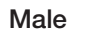

Female

Age at diagnosis, $y$

15-20

21-29

30-39

Race/ethnicity

Non-Hispanic white

Black

Hispanic

Other

\section{Education $^{a}$}

$<$ High school

High school graduate

Some college

College grad (2-y to 4-y college)

>Bachelor degree

Marital/relationship status ${ }^{a}$

Single/never married

Married/living as married

Divorced/separated

Currently in treatment ${ }^{a}$

Yes

No

Type of cancer

Germ cell cancer

Hodgkin lymphoma

Non-Hodgkin lymphoma

Sarcoma

Acute lymphocytic leukemia

Type of treatment received ${ }^{b}$

Bone marrow transplantation

Chemotherapy

Radiation

Surgery

\section{No. of Patients}

$\begin{array}{ll}192 & 36.7 \\ 331 & 63.3 \\ & \\ 83 & 15.9 \\ 210 & 40.2 \\ 230 & 44 \\ & \\ 310 & 59.3 \\ 45 & 8.6 \\ 108 & 20.7 \\ 60 & 11.5\end{array}$

11.5

$58 \quad 11.1$

$91 \quad 17.4$

$138 \quad 26.4$

$184 \quad 35.2$

$\begin{array}{ll}51 & 9.8\end{array}$

$273 \quad 52.2$

$219 \quad 41.9$

$30 \quad 5.7$

$91 \quad 17.4$

$419 \quad 80.1$

$204 \quad 39$

$142 \quad 27.2$

$131 \quad 25$

$25 \quad 4.8$

$21 \quad 4$

$16 \quad 3.2$

$363 \quad 74.4$

$172 \quad 35.2$

$204 \quad 40.2$

${ }^{a}$ Numbers do not always sum to total because of missing data.

${ }^{\mathrm{b}}$ Survivors can be counted more than once, depending on types of treatment received.

patients $(P<.001)$ were less likely to respond than nonHispanic white patients. ${ }^{20}$ Responses also varied by cancer site $(P<.04)$ from $38 \%$ of ALL and sarcoma survivors to $51 \%$ for HL survivors.

\section{Negative Life Impact}

Table 2 indicates the frequency with which respondents in the different developmental age groups perceived that their cancer experience had a negative impact on specific aspects of their life. With modest differences between the 
Table 2. The Percentage of Adolescent and Young Adult Cancer Patients in the Adolescent and Young Adult Health Outcomes and Patient Experience Study Reporting Negative, Positive, and No Impact by Age Groupa

\section{No. of Patients (\%)}

Life Impact Item
Relationship with friends
Relationship with your spouse/
significant other
Relationship with your mother
Relationship with your father
Relationship with your brothers
or sisters
Relationship with your child/children
Plans for the future and goal setting
Plans for work
Plans for education
Plans for getting married
Plans for having children
Financial situation
Dating
Sexual function/intimate relations
Feelings about the appearance
of your body
Confidence in ability to take care
of your health
Control over your life
Spirituality and religious beliefs

\begin{tabular}{ccc}
\multicolumn{3}{c}{ Ages $15-20$ Years, $\mathrm{n}=83$} \\
Negative & Positive & No Impact \\
$13(16.9)$ & $37(48.1)$ & $27(35.1)$ \\
$10(24.4)$ & $25(61)$ & $6(14.6)$ \\
& & \\
$5(6.3)$ & $62(77.5)$ & $13(16.3)$ \\
$5(6.6)$ & $51(67.1)$ & $20(26.3)$ \\
$2(2.5)$ & $63(79.7)^{\mathrm{c}}$ & $14(17.7)^{\mathrm{b}}$ \\
& & \\
$2(20)^{\mathrm{b}}$ & $1(10)^{\mathrm{c}}$ & $7(70)^{\mathrm{b}}$ \\
$27(34.6)$ & $37(47.4)$ & $14(17.9)$ \\
$25(33.8)$ & $17(23)$ & $32(43.2)^{\mathrm{c}}$ \\
$28(35.4)^{\mathrm{c}}$ & $21(26.6)$ & $30(38)^{\mathrm{c}}$ \\
$8(19)$ & $5(11.9)$ & $29(69)$ \\
$28(52.8)$ & $5(9.4)$ & $20(37.7)$ \\
$36(51.4)^{\mathrm{c}}$ & $7(10)$ & $27(38.6)^{\mathrm{c}}$ \\
$22(36.7)$ & $5(8.3)$ & $33(55)$ \\
$19(40.4)^{\mathrm{b}}$ & $3(6.4)$ & $25(53.2)^{\mathrm{b}}$ \\
$50(62.5)$ & $7(8.8)$ & $23(28.8)$ \\
& & \\
$22(27.2)$ & $35(43.2)$ & $24(29.6)$ \\
$37(46.3)$ & $26(32.5)$ & $17(21.3)$ \\
$7(9.7)$ & $38(52.8)$ & $27(37.5)$
\end{tabular}

\begin{tabular}{cccccc} 
Ages $21-29$ Years, $\mathrm{n}=210$ & \multicolumn{3}{c}{ Ages $30-39$ Years, $\mathrm{n}=230$} \\
Negative & Positive & No Impact & Negative & Positive & No Impact \\
$30(14.9)$ & $122(60.4)$ & $50(24.8)$ & $20(8.8)$ & $141(62.4)$ & $65(28.8)$ \\
$38(23)$ & $97(58.8)$ & $30(18.2)$ & $35(17.9)$ & $135(69.2)^{\mathrm{b}}$ & $25(12.8)$ \\
& & & & & \\
$19(9.3)$ & $134(65.7)$ & $51(25)$ & $20(9.6)$ & $130(62.5)$ & $58(27.9)$ \\
$14(7.4)$ & $111(59)$ & $63(33.5)$ & $10(5.6)$ & $109(60.6)$ & $61(33.9)$ \\
$11(5.7)$ & $119(61.3)$ & $64(33)$ & $14(6.6)$ & $137(64.9)$ & $60(28.4)$ \\
& & & & & \\
$2(3.2)$ & $40(63.5)$ & $21(33.3)$ & $22(15.9)^{c}$ & $84(60.9)$ & $32(23.2)$ \\
$63(31.2)$ & $96(47.5)$ & $43(21.3)$ & $70(31.1)$ & $106(47.1)$ & $49(21.8)$ \\
$81(39.9)$ & $60(29.6)$ & $62(56.4)$ & $83(38.2)$ & $55(25.3)$ & $79(36.4)$ \\
$35(19.2)$ & $43(23.6)$ & $104(57.1)$ & $30(18)$ & $28(16.8)$ & $109(65.3)$ \\
$26(23.6)$ & $22(20)$ & $62(56.4)$ & $25(34.2)$ & $13(17.8)$ & $35(47.9)$ \\
$112(63.6)$ & $13(7.4)$ & $51(29)$ & $92(59.4)$ & $6(3.9)$ & $57(36.8)$ \\
$141(69.5)$ & $16(7.9)$ & $46(22.7)$ & $146(64.9)$ & $15(6.7)$ & $64(28.4)$ \\
$43(44.3)$ & $9(9.3)$ & $45(46.4)$ & $29(44.6)$ & $8(12.3)$ & $28(43.1)$ \\
$108(57.8)$ & $11(5.9)$ & $68(36.4)$ & $125(59)$ & $9(4.2)$ & $78(36.8)$ \\
$121(59.6)$ & $26(12.8)$ & $56(27.6)$ & $139(61.5)$ & $19(8.4)$ & $68(30.1)$ \\
$73(35.6)$ & $85(41.5)$ & $47(22.9)$ & $81(35.7)$ & $87(38.3)$ & $59(26)$ \\
$98(47.8)$ & $59(28.8)$ & $48(23.4)$ & $110(48.9)$ & $66(29.3)$ & $49(21.8)$ \\
$8(4.3)$ & $106(56.4)$ & $74(39.4)$ & $10(4.8)$ & $129(62)$ & $69(33.2)$ \\
& & & & &
\end{tabular}

${ }^{a}$ For each life impact item, there are different percentages of the sample for which the question does not apply. Thus, the frequencies for positive, negative, and no impact do not add up to the total sample size for each age group. Also, the percentage denominator excludes respondents who indicated that a life impact item did not apply.

${ }^{\mathrm{b}}$ Chi-square $P<.05$ compared with the group ages 21 to 29 years.

${ }^{\mathrm{c}}$ Chi-square $P<.01$ compared with the group ages 21 to 29 years.

age groups, the most prevalent areas of life impacted in a negative way were: financial, body image, control over life, work plans, relationship with spouse/significant other, and plans for having children. Approximately twothirds of the groups ages 21 to 29 years and ages 30 to 39 years reported a negative impact $(69.5 \%$ and $64.9 \%$, respectively) on their financial situation. This was significantly higher than the $51.4 \%$ of those ages 15 to 20 years who reported a negative impact in this area $(P<.01)$

The majority of AYAs in this study reported that cancer had a negative impact on the way they felt about the appearance of their body: $62.5 \%$ of the group ages 15 to 20 years, $59.6 \%$ of the group ages 21 to 29 years, and $61.5 \%$ of the group ages 30 to 39 years reported a negative impact in this area. Almost half of respondents (47.5\%; with consistency across the 3 age groups) reported a negative impact of cancer on control over their lives. Similarly, approximately one-third of AYAs in all 3 age groups (ages $15-20$ years, $33.8 \%$; ages $21-29$ years, $39.9 \%$; and ages $30-39$ years, $38.2 \%$ ) reported that their cancer had a negative impact on their plans for work. Approximately 25\% of the overall sample reported a negative impact on their relationship with spouse/significant other. In the group ages 15 to 20 years, almost $17 \%$ reported a negative impact on friendships. In addition, $>50 \%$ of the sample reported that their cancer had a negative impact on their plans for having children.

Other significant differences in negative impact items for the younger age group compared with the middle age group were in plans for education $35.4 \%$ in the group ages $15-20$ years vs $19.2 \%: P<.01)$ and sexual function/intimate relationships ( $40.4 \%$ in the group ages $15-20$ years vs $57.8 \% ; P<.01)$.

\section{Positive Life Impact}

Endorsement that the cancer experience had a positive impact on specific aspects of life was evident across the 3 age groups, particularly with regard to relationships, future plans/goals, and health competence (Table 2). More than $75 \%$ of respondents in the group ages 15 to 20 years reported a positive impact in their relationships with mothers $(77.5 \%)$ and siblings (79.7\%), and the latter was significantly greater than that observed in the group ages 21 to 29 years $(61.3 \% ; P<.01)$. Other relationships that were impacted in a positive way as a result of cancer in the group ages 15 to 20 years were their relationships with 
fathers $(67.1 \%)$ and friends (48.1\%). In the group ages 21 to 29 years, the prevalence of positive changes in relationships ranged from a high of $65.7 \%$ (relationship with mother) to a low of $58.8 \%$ (relationship with spouse/significant other), demonstrating consistency across the relationship items. With regard to the group ages 30 to 39 years, positive changes in a relationship with spouse/significant other was greater in the group ages 30 to 39 years compared with the group ages 21 to 29 years $(P<.05)$. There were no other significant differences between the age groups 21 to 29 years and 30 to 39 years.

With regard to future plans, all 3 age groups reported that cancer had a similar level (approximately $46 \%$ of the sample) of positive impact on plans for the future and goal setting. Other areas of positive impact worth highlighting are within the domain of health competence (ie, confidence in your ability to take care of your health). Almost half of the sample (43.2\%) in the group ages 15 to 20 years reported a positive impact in the domain of health competence; similarly, $41.5 \%$ of the group ages 21 to 29 years $38.3 \%$ of the group ages 30 to 39 years reported a positive impact in this domain. Finally, $>50 \%$ of the overall sample reported a positive impact on their spirituality and religious beliefs as a result of cancer.

\section{DISCUSSION}

The current findings represent the largest populationbased, multisite study to date examining the psychosocial impact of being diagnosed with cancer as an AYA. The most prevalent negative life domains AYAs with cancer reported were specific to future plans (financial situation, plans for having children, plans for working) as well as body appearance and sense of control over life. Consistent with other studies of adult cancer survivors, ${ }^{23,24}$ AYAs in this study also reported that cancer had a positive impact in specific areas of their life. These findings demonstrate the coexistence (in the aggregate) of negative and positive psychosocial aspects of cancer in AYAs. Our findings also draw attention to specific psychosocial areas that may necessitate support and services to help AYAs minimize the negative consequences of cancer while promoting or supporting positive aspects during an already profound time of developmental change and turmoil.

The financial burden of cancer can be significant for individuals and families of all ages. However, it is likely that this strain is even greater for a young individual who is at the beginning stages of work and vocational development. Not only are there financial strains related to the cost of treatment and loss of pay resulting from time off from work (or delaying entry into the workforce), but
AYAs have lower earnings ${ }^{25}$ and have the highest uninsurance rate of any age cohort. ${ }^{26}$ Thirty percent of young adults lack health insurance, compared with $17 \%$ of older adults (ages 30-64 years). ${ }^{27}$ In a recent survey, two-thirds of young adults who had inadequate health coverage said they had forgone needed health care because of cost, including deciding not to fill a prescription, not consulting a physician when sick, or skipping a recommended test or treatment. ${ }^{26}$ The cost of forgoing needed cancerrelated care could be dire for the AYA population.

Our findings support the limited research that has identified a negative relation between cancer and body image, ${ }^{16,28}$ supporting the need to address body image issues in the AYA population. Adolescence and young adulthood is a critical time of negotiating a healthy body image, and a diagnosis of cancer can exacerbate this already challenging developmental task. A poor body image can lead to low self-esteem and may affect the ability to form healthy peer and intimate relationships during young adulthood. ${ }^{29}$ Several interventions have been successful in improving body image of obese $\mathrm{AYAs}^{30}$ as well as survivors of adult cancers (breast and prostate), ${ }^{31}$ but we were not able to locate any body image interventions that were developed specifically for the AYA cancer population. This is an important area for future research.

Another highly prevalent area of negative impact reported by all 3 groups was control over life. Lower perceived control over one's life has been related to lower treatment adherence in the general adult cancer population. ${ }^{32}$ This finding is even more alarming, because adolescent patients are at the greatest risk of nonadherence to their cancer chemotherapy regimen. ${ }^{33}$ In addition, it has been established that greater perceived control over one's life and the course of illness improves quality-of-life outcomes in the AYA population. ${ }^{34}$ Clinicians working with AYAs should try to foster a sense of control over aspects of the environment that are within a patient's control, such as treatment adherence, follow-up care, and health-promoting activities (eg, healthy diet and physical activity), that may reduce risk of recurrence and also contribute to better quality of life in survivors of adult cancers. 35,36

Another important finding was that 1 in 3 teenage respondents indicated that cancer had disrupted their education. A diagnosis of cancer as an adolescent limits opportunities for educational or vocational advancement, ${ }^{37}$ which, in turn, can contribute to poorer health outcomes $^{38}$ and possibly may limit life-long economic or employment opportunities. In addition, the pediatric literature suggests that time away from school as a result of treatment can have acute effects, such as a depression, 
poor self-esteem, and lack of interest, as well as more long-term effects, such as loss of purpose or difficulty re-engaging in educational pursuits. ${ }^{39}$ Thus. this finding underscores the importance of finding ways to help younger AYAs stay engaged and plan for and attain their educational goals.

With regard to the negative impact of cancer on relationships, 1 in 4 teens (ages 15-20 years) reported that cancer had a negative impact on their relationship with their significant other. Furthermore, among young adults, 1 in 4 survivors ages 21 to 29 years and 1 in 5 survivors ages 30 to 39 years also reported a negative impact in this area. In the general population, poor relationships have been related to distress and poor quality of life ${ }^{40}$ and likely have implications for social functioning in general.

Over $50 \%$ of survivors in all 3 age groups reported that cancer has had a negative impact on their "plans for having children." With the possibility of infertility because of treatments like chemotherapy, radiation, and certain types of surgery, the American Society of Clinical Oncology consensus statement ${ }^{41}$ indicates that oncologists should discuss fertility preservation options at the time of diagnosis and should refer patients to appropriate reproductive resources. Research has demonstrated that this topic is highly important to AYAs, ${ }^{18}$ yet barriers to fertility preservation persist, and many physicians are reluctant to bring up this topic, often because of the fear of worrying their patients about the expense of egg and sperm storage or the lack of appropriate resources and guides to help their patients. ${ }^{42}$ We also observed a significant impact on sexual function and intimate relationships in the younger group compared with the middle group, underscoring the importance of this understudied topic in survivorship research, particularly among AYAs.

In our study, a notable percentage of AYAs reported positive changes resulting from their cancer experience. Positive changes in relationships with different family members and friends were prevalent across the 3 age groups. This finding is highly salient when examined within the context of a previous study in which support from family and friends were identified as the most highly ranked supportive care need of the AYA group. ${ }^{18}$ The broader social support literature also suggests improved health outcomes and better adjustment in AYAs with cancer who report high levels of social support. ${ }^{43}$

Some AYAs reported positive changes in plans for their future as well as confidence in their ability to take care of their health. With respect to future planning, a diagnosis of cancer can lead to focused attention toward an individual's future goals and purpose, ${ }^{23}$ particularly for younger adults who have a longer time horizon and have more opportunities to achieve desired goals. It is not uncommon for young adults with serious illnesses, including cancer, to re-examine aspects of their lives, such as work, education, and family, and make changes to progress to a new or better life. ${ }^{44}$ The positive impact on their confidence in taking care of their health is important, because AYAs with cancer likely will be managing and monitoring their health for many years, and health competence has been positively associated with emotional, physical, social, and family well being in survivors of other cancers. ${ }^{45}$ With the potential for recurrence or late or long-term side effects associated with their disease, it is heartening that approximately $40 \%$ of AYAs in the sample felt more confident. However, it is concerning that approximately $33 \%$ reported a negative impact on confidence in their ability to take care of their health. Future research is needed to better understand the factors that contribute to reporting more negative, as well as more positive, experiences, and to develop better interventions that prevent negative sequelae and promote positive adaptation and potential for personal growth.

This study has certain limitations. First, the lack of a control group limited our ability to disentangle which elements were developmental and which elements were because of cancer. The mostly uniform psychosocial impact of cancer across the 3 age groups provided some evidence that the impact had more to do with the cancer experience than development. Second, our life impact checklist was not designed to be used as a scale, which limited our ability to examine antecedents or outcomes of life impact. The measure also precluded our ability to determine whether cancer had both a positive impact and a negative impact within specific impact items. For example, cancer may have had a negative impact on some friendships but a positive impact on others. The measure was developed as a general gauge of attitude or perception of how cancer affected one's life. However, because of the lack of research in this area, our intention was to examine the breadth of psychosocial domains to identify areas for future research and follow-up. Third, the requirement that participants speak English as well as our overall response rate of $43 \%$ limits the generalizibility of our findings. Finally, as mentioned above, there was a bias toward nonparticipation by men, Hispanics, and nonHispanic blacks, which also limits the generalizibility of our study.

This population-based, multicenter study significantly contributes to the sparse body of research on the psychosocial impact of being diagnosed with cancer as an 
AYA. To our knowledge, this study is the first to examine the negative and positive impact in this population, offering a more comprehensive psychosocial understanding of this group. Findings from this study suggest, with few differences, that cancer has a uniformly negative and positive impact on AYAs across the different developmental periods. Taking both deficit-based and strength-based approaches to understanding the impact of cancer in the AYA population can allow us to focus research and interventions on minimizing the negative consequences of cancer while promoting the positive aspects in AYA cancer survivors. In addition, future research might focus on understanding the characteristics of individuals who report a positive impact, so that we can use such information to intervene with AYAs who may be struggling in these areas.

\section{FUNDING SOURCES}

This work was supported by N01PC-2010-00032 (University of Iowa), N01PC-2010-00140 (Cancer Prevention Institute of California), N01PC-2010-00034 (Public Health Institute), N01PC-2010-00035 (University of Southern California), N01PC-2010-00029 (Fred Hutchinson Cancer Research Center), N01PC-2010-00028 (Wayne State University), and N01PC-2010-00030 (Louisiana State University Health Sciences Center).

\section{CONFLICT OF INTEREST DISCLOSURES}

The authors made no disclosures.

\section{REFERENCES}

1. Havighurst RJ. Developmental Tasks and Education. New York: David McKay; 1952.

2. Levinson D. The Seasons of a Man's Life. New York: Ballantine; 1978.

3. Suris JC, Michaud PA, Viner R. The adolescent with a chronic condition. Part I: developmental issues. Arch Dis Child. 2004;89:938942.

4. Rowland JH. Developmental Stage and Adaptation: Adult Model. New York: Oxford University Press; 1990.

5. Bellizzi KM. Expressions of generativity and posttraumatic growth in adult cancer survivors. Int J Aging Hum Dev. 2004;58:267-287.

6. Barakat LP, Alderfer MA, Kazak AE. Post-traumatic growth in adolescent survivors of cancer and their mothers and fathers. J Pediatr Psychol. 2004;31:413-419.

7. Bellizzi KM, Miller MF, Arora NK, Rowland J. Positive and negative life changes experienced by survivors of non-Hodgkin's lymphoma. Ann Behav Med. 2007;34:188-199.

8. Zebrack BJ, Stuber ML, Meeske KA, et al. Perceived positive impact of cancer among long-term survivors of childhood cancer: a report from the childhood cancer survivor study [published online ahead of print March 22, 2011]. Psychooncology. 2011.

9. Nagarajan R, Neglia JP, Clohisy DR, et al. Education, employment, insurance, and marital status among 694 survivors of pediatric lower extremity bone tumors: a report from the Childhood Cancer Survivor Study. Cancer. 2003;97:2554-2564.

10. Lee YL, Santacrocec SJ. Posttraumatic stress in long-term young adult survivors of childhood cancer: a questionnaire survey. Int J Nurs Studies. 2007;44:1406-1417.
11. Mellon S, Northouse LL. Family survivorship and quality of life following a cancer diagnosis. Res Nurs Health. 2001;24:446-459.

12. Albritton K, Caligiuri M, Anderson B, et al. Closing the Gap: Research and Care Imperatives for Adolescents and Young Adults With Cancer: Report of the Adolescent and Young Adult Oncology Progress Review Group. Washington, DC: US Department of Health and Human Services, National Institutes of Health, National Cancer Institute, LiveStrong Young Adult Alliance; 2006.

13. Burke ME, Albritton K, Marina N. Challenges in the recruitment of adolescents and young adults to cancer clinical trials. Cancer. 2007; 110:2385-2393.

14. Bleyer A, Budd T, Montello M. Adolescents and young adults with cancer: the scope of the problem and criticality of clinical trials. Cancer. 2006;107:1645-1655.

15. Tricoli JV, Seibel NL, Blair DG, Albritton K, Hayes-Lattin B. Unique characteristics of adolescent and young adult acute lymphoblastic leukemia, breast cancer, and colon cancer. J Natl Cancer Inst. 2011;103:628-635.

16. Pendley JS, Dahlquist LM, Dreyer Z. Body image and psychosocial adjustment in adolescent cancer survivors. J Pediatr Psychol. 1997;22:29-43.

17. Zebrack B, Bleyer A, Albritton K, Medearis S, Tang J. Assessing the health care needs of adolescent and young adult (AYA) cancer patients and survivors. Cancer. 2006;107:2915-2923.

18. Zebrack BJ, Mills J, Weitzman TS. Health and supportive care needs of young adult cancer patients and survivors. J Cancer Surviv. 2007;1:137-145.

19. Kyngas H, Mikkonen R, Nousiainen EM, et al. Coping with the onset of cancer: coping strategies and resources of young people with cancer. Eur J Cancer Care. 2001;10:6-11.

20. Harlan LC, Lynch CF, Keegan TH, et al; AYA HOPE Study Collaborative Group. Recruitment and follow-up of adolescent and young adult cancer survivors: the AYA HOPE Study. J Cancer Surviv. 2011;5:305-314.

21. Ganz P, Desmond K, Leedham B, Rowland J, Meyerowitz B, Belin T. Quality of life in long-term, disease-free survivors of breast cancer: a follow-up study. J Natl Cancer Inst. 2002;94:39-49.

22. Zebrack BJ. Developing a new instrument to assess the impact of cancer in young adult survivors of childhood cancer. $J$ Cancer Surviv. 2009;3:174-180.

23. Bellizzi KM, Blank TO. Understanding the dynamics of post-traumatic growth in breast cancer survivors. Health Psychol. 2006;25:4756.

24. Stanton AL, Bower JE, Low CA.Posttraumatic growth after cancer. In:Calhoun LG,Tedeschi RG, eds. Handbook of Post-Traumatic Growth: Research and Practice. Mahwah, NJ: Erlbaum; 2006:138175.

25. US Department of Labor Statistics. Differences in Earnings by Age and Sex in 2005. Available at: http://www.bls.gov/opub/ted/2006/ oct/wk1/art01.htm. Accessed June 2, 2011.

26. Nicholson JL, Collins SR, Mahato B, et al. Rite of Passage? Why Young Adults Become Uninsured and How New Policies Can Help. Washington, DC: The Commonwealth Fund; 2009.

27. Schwartz K, Schwartz T. Uninsured Young Adults: A Profile and Overview of Coverage Options. Washington, DC: Kaiser Commission on Medicaid and the Uninsured; 2008.

28. Carpentier MY, Fortenberry JD. Romantic and sexual relationships, body image, and fertility in adolescent and young adult testicular cancer survivors: a review of the literature. $J$ Adolesc Health. 2010;47:115-125.

29. Davison TE, McCabe MP. Adolescent body image and psychosocial functioning. J Soc Psychol. 2006;146:15-30.

30. Huang JS, Norman GJ, Zabinski MF, Calfas K, Patrick K. Body image and self-esteem among adolescents undergoing an intervention targeting dietary and physical activity behaviors. I Adolesc Health. 2007;40:245-251.

31. Harrington JM, Badger TA. Body image and quality of life in men with prostate cancer [serial online]. Cancer Nurs. 2009;32:E1-E7.

32. Partridge AH, Avorn J, Wang PS, Winer EP. Adherence to therapy with oral antineoplastic agents. J Natl Cancer Inst. 2002;94:652661. 
33. Festa RS, Tamaroff MH, Chasalow F, Lanzkowsky P. Therapeutic adherence to oral medication regimens by adolescents with cancer. I. Laboratory assessment. J Pediatr Psychol. 1992;120:807-811.

34. Sorgen KE, Manne SL. Coping in children with cancer: examining the goodness-of-fit hypothesis. Child Health Care. 2002;31:191-207.

35. Holmes MD, Chen WY, Feskanich D, Kroenke CH, Colditz GA. Physical activity and survival after breast cancer diagnosis. JAMA. 2005;293:2479-2486.

36. Bellizzi KM, Rowland J, Arora N, Hamilton AS, Miller MF, Aziz NM. Physical activity and quality of life of adult survivors of nonHodgkin's lymphoma. J Clin Oncol. 2009;2007:960-966.

37. Maslow GR, Haydon AA, Ford CA, Halpern CT. Young adult outcomes of children growing up with chronic illness: an analysis of the National Longitudinal Study of Adolescent Health. Arch Pediatr Adolesc Med. 2011;165:256-261.

38. Zebrack BJ, Gurney JG, Oeffinger K, et al. Psychological outcomes in long-term survivors of childhood brain cancer: a report from the Childhood Cancer Survivor Study. J Clin Oncol. 2004;22:9991006.

39. Vance $\mathrm{YH}$, Eiser C. The school experience of the child with cancer. Child Care Health Dev. 2002;1:5-19.
40. Myers DG.Close relationships and quality of life. In:Kahneman D,Diener E,Schwarz N, eds. Well-Being: The Foundations of Hedonic Psychology. Vol xii. New York: Russell Sage Foundation; 1999:374-391.

41. Lee SJ, Schover LR, Partridge AH, et al. American Society of Clinical Oncology recommendations on fertility preservation in cancer patients. J Clin Oncol. 2006;24:2917-2931.

42. Quinn GP, Vadaparampil ST; Fertility Preservation Research Group. Fertility preservation and adolescent/young adult cancer patients: physician communication challenges [serial online]. $J$ Adolesc Health. 2009;44:394-400.

43. Manne S, Miller D. Social support, social conflict, and adjustment among adolescents with cancer. J Pediatr Psychol. 1998;23:121-130.

44. Devine KA, Reed-Knight B, Loiselle KA, Fenton N, Blount RL. Posttraumatic growth in young adults who experienced serious childhood illness: a mixed-methods approach. J Clin Psychol Med Settings. 2010;17:340-348.

45. Arora NK, Johnson P, Gustafson DH, McTavish F, Hawkins RP, Pingree S. Barriers to information access, perceived health competence, and psychosocial health outcomes: test of a mediation model in a breast cancer sample. Patient Educ Couns. 2002;47:37-46. 